\title{
Musculoeskeletal disorder related to the work of doctors who perform medical invasive evaluation
}

\author{
D.P. Maciel, R.A.M. Millen, C.A. Xavier, L.C. Morrone and J.S. Silva-Júnior ${ }^{*}$ \\ Deparment of Social Medicine, Santa Casa Medical Sciences School, Rua Dr. Cesário Mota Junior, 61 - $6^{\circ}$ andar, \\ 01221-020, São Paulo, SP, Brasil, fax: (55 11) 3222-0412
}

\begin{abstract}
Background: Experts in medical invasive evaluations, like colonoscopy, could be exposed to ergonomic risks during their work. Little attention has been given to these spectrum of occupational health. Its aimed to analyze possible clinical problems related to upper limb of physicians who perform those exams. Methods: Cross-sectional study in a health service in Sao Paulo, Brazil. For evaluation of the workplace of the colonoscopist physician were applied two tools: Couto's check list and Sue Rodgers Method. The results direct the potential risk to upper limb injuries. Results: The procedure is done and the final report is issued. There are no mandatory pauses during workday. The dominant hand holds the tube insertion with pincer movement during the exam, digital prehension being necessary at times. For this activity the employee has to use some strength. Couto's check list indicates a significant biomechanical factor. Sue Rodgers's Method states high ergonomic risk for dominant hand/wrist/fingers. Conclusion: The main risk for the development of health disorders are associated with the use of force and repetitive movements of hands. The adoption of regular breaks to recover most of the structures required is recommended.
\end{abstract}

Keywords: colonoscopy, cumulative trauma disorders, occupational health, healthcare professional, ergonomics

\section{Introduction}

The social transformations generated by the Industrial Revolution occurred in England in the nineteenth century led profound changes in the process and organization of work, culminating in emergence of numerous cases of occupational diseases. In Brazil, musculoskeletal diseases (MSD) caused by static and dynamic overload become more numerous from 1980 's, when they began to be described the first cases of tenosynovitis in typists.

The main areas of the body affected by workrelated musculoskeletal injuries include lower back and upper limbs. Repetitive movements, sustained and static postures of muscles are causes of these disorders. Evidence suggests that doctors in the practice of their profession are also exposed to workrelated musculoskeletal sickness factors as result of pattern of movement executed during their activies. [6]
Much has been discussed about the safety of patients during invasive diagnostic procedures such as endoscopy, colonoscopy and cholangiopancreatography, but less attention has been directed to occupational health of doctors who perform that. Studies have estimated a prevalence of musculoskeletal pain ranging from $37 \%$ to $89 \%$ in medical endoscopists. [1] The risk of developing MSD among endoscopists is associated with performing repetitive hand movements and support postures during the examination. [6]

Endoscopy is a procedure of short duration, with negligible impact of the prolonged use of force by the endoscopist. In this exam the most important factor to prevent musculoskeletal injuries is to maintain the neutral stance of the body, avoiding the overhead static. However, the performance of colonoscopy involves repetitive movements with force being taken for a longer duration, also associated with prolonged maintenance of awkward postures. [1]

${ }^{*}$ Corresponding author. E-mail: silvajunior.js@gmail.com. 
During the examination, the left hand holds and stabilizes the control of the endoscope and the left thumb manipulates control knobs. Besides the left thumb, the fingers of left hand promote additional strength to control equipment manipulation. The right hand holds the insertion tube doing a pincer grip and hand prehension. The progression of tube during exam is made by straight push, pull and circular movements of dominant hand, i.e. right hand in most of the times, with the aid of dominant arm. The application of circular movements on the insertion tube allows greater range of motion of the tip of the device. These activities require extensive use of the wrist in flexion/extension and ulnar/radial deviation. In addition, the right hand is also used for the insertion of the acessories for collect material to biopsy. The high strength by fingers, associated with the wrist postures, can increase the risk of musculoskeletal injuries. [1]

It's proposed to assess ergonomics factors in work of the doctors responsible for conducting the colonoscopy procedures to recommend improvements in working condition. Thus, these can promote a reduction of fatigue and musculoskeletal injuries. Consequently it leads to job satisfaction and increased productivity.

The aim of this study is an ergonomic assessment of workplace colonoscopy during the examination in an outpatient setting.

\section{Method}

The study was carried out at a central of colonoscopy at a laboratory in the city of São Paulo, Brazil. The patients were cared at outpatient clinics, aged between 15 and 65 years for that kind of exam.

Data collection was initiated by an assessment of the workplace with regard to room size and spatial distribution of objects/furniture. The height, distance and placement of these were measured with a metric tape.

The authors applied two ergonomic tools for assessing the potential risk for the development / worsening of musculoskeletal disorders, which were Couto's Checklist for upper limbs and Sue Rodgers Method. These tools were applied for the evaluation of six consecutive cycles of work performed in a regular day.

The Couto's checklist [3] evaluates 6 items related to work environment: (1) physical overload, (2) force by hands, (3) working postures, (4) job, (5) repeata- bility and work organization; (6) tool of work. For each item there are questions that are scored as 0 or 1. The final sum of points defines the degree of risk for upper limb injuries: 0 to 3 points - the absence of biomechanical factors; 4 to 6 points - biomechanical factor negligible; 7 to 9 points - moderate biomechanical factor; 10 to 14 points - significant biomechanical factor; 15 or more points - very significant biomechanical factor.

The Sue Rodgers Method [5] evaluates observed body segments by analyzing its effort to develop the activity as well as the amount that this effort is present within a work cycle. In this study the duty cycle is over, then we considered a cycle as the total time to perform an examination. The maneuvers required for the progression of the device and the position taken was used as a basis for analyzing the amount of work done during the cycle. The study results show the degree of risk for injury to muscle groups and suggests priorities for ergonomic intervention.

\section{Results}

\subsection{Evaluation of workstation}

The exam room has dimensions of $4.2 \times 3.7$ meters. In this sector, two people working: the doctor responsible for performing the tests and a nursing assistant. The examination table is located in front of and parallel to the worker. It is $94 \mathrm{~cm}$ height. The rack and colonoscope monitor are located after the examination stretcher, against the wall. The rack height is $1.26 \mathrm{~m}$ and the monitor is located $20 \mathrm{~cm}$ from the surface of the rack. The monitor has a 43 $\mathrm{cm}$ wide and $35 \mathrm{~cm}$ high.

The device consists of a rubber insertion tube, 12.8 $\mathrm{mm}$ diameter and $1.60 \mathrm{~cm}$ length, $7 \mathrm{~cm}$ handle command diameter, two control systems for movements up / down and left / right. This handle is moved continuously during the examination by the thumb and left hand of the doctor.

\subsection{Organization of work}

The exams are scheduled every 30 minutes, totaling 15 tests per day. We evaluated six consecutive cycles. The duty cycle was divided between the examination and typing report, with time for each activity timed according to Table 01 . 
Table 01. Time to perform doctor's activities during work cycles evaluated in colonoscopy exams, São Paulo, 2011

\begin{tabular}{ccc} 
Cycle & Time of exam & Time to type \\
\hline 1 & $10: 10 \mathrm{~min}$ & $02: 26 \mathrm{~min}$ \\
2 & $07: 13 \mathrm{~min}$ & $01: 36 \mathrm{~min}$ \\
3 & $16: 03 \mathrm{~min}$ & $03: 42 \mathrm{~min}$ \\
4 & $12: 55 \mathrm{~min}$ & $02: 23 \mathrm{~min}$ \\
5 & $17: 14 \mathrm{~min}$ & $02: 30 \mathrm{~min}$ \\
6 & $26: 00 \mathrm{~min}$ & $03: 51 \mathrm{~min}$ \\
\hline Mean time & $\mathbf{1 9 : 2 2} \mathbf{m i n}$ & $\mathbf{0 2 : 5 1} \mathbf{~ m i n}$ \\
\hline
\end{tabular}

While performing colonoscopy workers are standing. There are no breaks between scheduled appointments. They pause between the patients. The monitor is located at a distance of $70 \mathrm{~cm}$ of the worker, lateralized to the right and the center at a distance of $1.62 \mathrm{~cm}$ from the ground. There isn't adjustment of monitor height, but there is possibility of lateral movement and direction up / down, allowing change of distance and lateral positioning of the monitor in relation to the operator.

The movements performed by the arms, wrists / hands during insertion and removal of the apparatus can be divided over the right and left.

The left arm holds the handle in solid support of the device, with forearm flexion over $90^{\circ}$ and abduction of the arm in $30^{\circ}$. The left hand is responsible for moving the control handles of the insertion tube to achieve the movements of the tip of the device up / down and lateralization left / right. Moreover, ocasionally moves the entire handle up and down allowing a range of motion of the tip of the device. The left hand holds the wrist flexion and extension maneuvers forced to an angle of 30 degrees.

The right arm is responsible for handling the insertion tube during the examination. The movements include flexion, pronation / supination and circular. The right hand holds the tube insertion pincer pulp throughout the examination. At times, the right hand holds the tube in prehension when is necessary increases strength to carry out the circular movement. The right hand performance is the most important for the insertion of the device.

The right hand is also used to insert fittings and clamps for perfoming procedures such as biopsies and remove polyps.
The worker stands throughout the test, being allowed to adopt postures of comfort and mobilization of the legs. Throughout the examination photographic recording in its own system of capture is necessary. This registration is done by pushing the pedal with the left foot. On average, about 28 records are made per exam throughout the procedure.

The activity of the test is interspersed with the typing of the report. This activity lasts approximately 2 minutes and 51 seconds, taking place at the workplace next to the examination table.

\subsection{Ergonomic tools application}

\subsubsection{Couto's checklist}

The application of this checklist to study impacts to upper limb overload sum 11 points, which means a significant biomechanical factor. Criteria that scored negatively refer to the use of force with the hands, particularly in clamp position during most of the cycle. Also stood out in a negative way to maintain a static position of the upper and forced movements of flexion / extension wrist.

\subsubsection{Sue Rodgers method}

By applying this method a high risk of musculoskeletal injury in the upper limbs was shown, mainly related to right wrist, hand and fingers. A moderate burden was detected to both arms and shoulders, besides left wrist.

\section{Discussion}

For ergonomic evaluation performed on the job colonoscopy, the occurrence of ergonomic risks by awkward postures and repetitive movements with force was observed and confirmed through the application of ergonomic tools.

The results of the checklist allowed the identification of the joint in major occupational risk by ergonomic factors, represented by the wrist / hand / fingers of the right arm. Performance os therm during exam raises the overall risk potential of the position evaluated.

The main effort identified and which causes the elevation of the potential risk of injury is the maintenance of the pincer grip of the right thumb during the entire procedure. This pincer movement is used to power during the progression of the insertion tube through intestines. Shergill et al. [2] evaluated this directly through muscular electromyography of the 
thumb during the colonoscopy. The move requires strength of circular movement during insertion of the device and during adjustment maneuvers.

The left wrist extensor muscles are also potentially at risk of injury. It's because the left hand is used for support and stabilization of control commands. Also the left thumb held constant and repetitive movement during manipulation of the handles. Shergill et al. [6] evaluated the muscle strength of the extensor muscles of the left wrist and right wrist during the maneuvers for insertion and withdrawal of the colonoscope. These authors observed that the peak muscle strength during insertion of the device exceeds the limit proposed by American Conference of Industrial Hygienists (ACGIH).

Despite the high potential of identified ergonomic risk for the wrists / hands / fingers, the options to minimize overhead in these joints are limited. These movements are essential in the procedure, both at the time of insertion and the removal of the device. Orientation about risk awareness must be done to worker adopts more appropriated postures that decrease potential for musculoskeletal injury. This is an important intervention.

Furthermore, additional information to recognize the occurrence of muscle fatigue of the hand and forearm reduces the potential severity of these lesions. [1] Another way to reduce occurrence of injuries is the adoption of a break between exams scheduled that promotes musculoskeletal system recovery. In the present study, there is a non-scheduled break of about 7 minutes. However, this cycle time work could be insufficient for workers with less experience, requiring a longer time for the exam. These workers need a more flexible schedule that allows the maintenance of breaks.

The ergonomic optimization of the colonoscopy room is obtained by better distribution of furniture, height adjustment of the monitor and the examination table. The main determinants in the posture of the body are related to the location of the patient, colonoscopy equipment and monitor. The location of the monitor is the most important determinant in the posture of the trunk and head. Monitors should be located in front of the worker and the adjustable visibility to keep the neck in a neutral position and prevent rotation and flexion of the spine. [1]
The distance from the monitor to work depends on screen size, clarity of image and preference of the worker. Studies based on laparoscopy recommend the optimal distance to the examiner of the display between 52 and $182 \mathrm{~cm}$. [1] In the present study, the distance from the monitor to the worker is $70 \mathrm{~cm}$, which is considered a suitable distance and allows the test without flexing the lumbar spine for better image clarity. However, the monitor is lateralized to the right relative to the position of the worker, which leads to lateral rotation of the cervical spine during the procedure. Thus there is a predisposition to cervical and back pain.

\section{Conclusion}

In the ergonomic evaluation performed on the workplace of physicians who perform colonoscopy exams, the main risk for the development health disorders are associated with the use of force and repetitive movements of hands. This can be caused by the anti-ergonomic situation of making a pincer movement during almost the entire work cycle. The adoption of regular breaks to recover most of the structures required is recommended.

\section{References}

[1] A.K. Shergill et al, Ergonomics and GI endoscopy, Gastrointestinal endoscopy, 2009, v. 70, n. 1, pp. $145-153$.

[2] A.K. Shergill et al, Pinch force and forearm muscle load during colonoscopy: a pilot study, Gastrointest endosc, 2009, v. 69 , pp. $142-146$.

[3] H.A. Couto, Ergonomia aplicada ao trabalho: manual técnico da máquina humana, ed. Ergo, Belo Horizonte, 1995.

[4] M.N. Appleyard et al, The measurement of forces exerted uring colonoscopy, Gastrointest endoscopy, 2000, v. 52, pp. 237-40.

[5] S. Rodgers, Functional job evaluation technique in Ergonomics, Occupational Medicine: State of the Art Reviews, 1992, v. 7, pp. 679-711.

[6] S. O. Bkin et al, Musculoskeletal injuries among ERCP endoscopists in Canada, Can J Gastroenterol, 2002, v. 16, n. 6, pp. $369-374$.

[7] S.R. Faraco, Pericias em DORT, ed. LTr, São Paulo, 2010. 